\title{
Programa de atenção nutricional: marco histórico na política pública para pessoas com necessidades alimentares especiais no Município de Curitiba, Paraná
}

\author{
Nutritional care program: historic landmark in public policy for people with special dietary \\ needs in the City of Curitiba, Parana State, Brazil
}

Patrícia Audrey Reis Gonçalves Pinheiro' Angela Cristina Lucas de Oliveiral Karyne Sant'ana Gonzales Gomes² Caryna Eurich Mazur ${ }^{3}$ Maria Eliana Madalozzo Schieferdecker ${ }^{4}$

1 Departamento de Atenção Primária à Saúde / Coordenação do Cuidado. Secretaria Municipal da Saúde de Curitiba. Curitiba, PR, Brasil.

${ }^{2}$ Distrito Sanitário Santa Felicidade / Núcleo de Apoio à Saúde da Família. Secretaria Municipal da Saúde de Curitiba. Curitiba, PR, Brasil.

${ }^{3}$ Programa de Pós-graduação em Segurança Alimentar e Nutricional. Universidade Federal do Paraná. Curitiba, PR, Brasil.

${ }^{4}$ Departamento de Nutrição e do Programa de Pósgraduação em Segurança Alimentar e Nutricional. Setor de Ciências da Saúde. Universidade Federal do Paraná. Curitiba, PR, Brasil.

Correspondência / Correspondence Maria Eliana M. Schieferdecker UFPR - Departamento de Nutrição Av. Prefeito Lothário Meissner, 632 - Jd. Botânico 80210-170 Curitiba, PR, Brasil

E-mail:meliana@ufpr.br

\section{Resumo}

Este trabalho objetiva traçar o marco histórico do Programa de Atenção Nutricional às Pessoas com Necessidades Especiais de Alimentação (PAN) e os avanços e desafios no que diz respeito à política pública para pessoas com necessidades alimentares especiais no município de Curitiba, Paraná. Historicamente, o PAN foi criado com o intuito de substituir o antigo Programa de Dietas Enterais e Leites Especiais e organizar a atenção nutricional no município, a fim de oferecer melhor assistência nutricional a essas pessoas, com ações baseadas nos princípios do Sistema Único de Saúde (SUS). Nessa perspectiva, buscou-se pontuar o marco histórico do PAN na gestão das políticas públicas dentro do SUS, e diante de tal marco, o impacto na gestão, nos profissionais e usuários envolvidos no processo.

Palavras-chave: Terapia Nutricional. Política Pública. Políticas Públicas de Saúde. Pessoas com Deficiência. Direitos Humanos. Sistema Único de Saúde. 


\section{Abstract}

This paper aims to draw the historical framework of the Nutritional Care Program for People with Special Dietary Needs (PAN), the advancements and challenges in public policy for people with special dietary needs in the city of Curitiba, Parana state, Brazil. Historically, PAN was created to replace the former Enteral Diets and Special Milks Program and to organize nutritional care in the city, so as to offer better nutritional care to these people, based on the principles of the Unified Health System (SUS). In this perspective, we attempted to draw the historical framework of PAN in the management of public policies within the SUS, and at such a landmark, the impact in management, professionals and users involved in this process.

Key words: Nutrition Therapy. Public Policy. Health Public Policy. Disabled People. Human Rights. Unified Health System.

\section{Introdução}

Alimentação e nutrição são condicionantes da saúde e da vida humana. Nos planos individual e coletivo, esses atributos estão assinalados na Declaração Universal dos Direitos Humanos, promulgada há 50 anos, reafirmada no Pacto Internacional sobre Direitos Econômicos, Sociais e Culturais (1966) e incorporada à legislação nacional em 1992.1.3

O Direito Humano à Alimentação Adequada (DHAA) é o direito inerente a todas as pessoas de ter acesso regular, permanente e irrestrito, quer diretamente ou por meio de benefícios financeiros, a alimentos seguros e saudáveis, em quantidade e qualidade adequadas e suficientes, correspondentes às tradições culturais do seu povo e que garantam a vida livre do medo, digna e plena nas dimensões física e mental, individual e coletiva. ${ }^{4}$

A terapia nutricional (TN) baseada na garantia do DHAA objetiva o adequado fornecimento de nutrientes necessários para o funcionamento do organismo e visa ainda manter ou recuperar o bom estado nutricional, reduzir o risco de complicações, promover rápida recuperação, reduzir o tempo de hospitalização e reduzir a morbimortalidade. A terapia nutricional domiciliar (TND) é uma modalidade de atendimento nutricional, relacionada à assistência domiciliar, que busca melhorar a qualidade de vida do indivíduo, com melhora de seu prognóstico do quadro clínico associado ao convívio social e familiar. ${ }^{5,6}$ 
Os indivíduos com necessidades alimentares especiais relacionadas às vias alternativas de alimentação também devem ter seu DHAA garantido por meio de políticas públicas. A partir da implementação dessas políticas, a atenção nutricional será concebida como parte do cuidado integral na Rede de Atenção à Saúde (RAS), tendo a atenção básica como coordenadora do cuidado e ordenadora da rede. ${ }^{7}$

Dessa maneira, o trabalho objetiva traçar o marco histórico do Programa de Atenção Nutricional às Pessoas com Necessidades Especiais de Alimentação (PAN), no município de Curitiba, Paraná, descrevendo os avanços e desafios no que diz respeito à política pública para pessoas com necessidades alimentares especiais.

\section{Método}

Foi realizado levantamento de dados históricos a partir da implementação do atendimento às pessoas com necessidades especiais de alimentação em 2002. Os dados foram obtidos por meio de planilhas em meio eletrônico, dos usuários acompanhados pelo PAN, e documentos oficiais da Secretaria Municipal de Saúde (SMS), da Prefeitura Municipal de Curitiba (PMC).

\section{Resultados}

\section{Histórico do Programa de Atenção Nutricional (2002 a 2006)}

A legislação vigente para a TN é a Resolução da Diretoria Colegiada (RDC) n 63, que estabelece o Regulamento Técnico para a TN no âmbito hospitalar e serviços credenciados pelo SUS, e a Portaria do Ministério da Saúde n ${ }^{\circ} 120$, que também normatiza a TN. ${ }^{5,8}$

A partir de 2002, a SMS de Curitiba começou a constatar a demanda de solicitações de fórmulas alimentares industrializadas (FAI), tais como suplementos, fórmulas completas e fórmulas infantis para situações especiais. Nesse período, os pedidos de FAI eram aceitos conforme as prescrições vindas dos hospitais e dos ambulatórios de serviços especializados, e a aquisição das fórmulas ocorria por meio de compra direta, por profissional administrativo, sem a padronização dos produtos.

No entanto, devido à crescente demanda para o atendimento de pacientes que necessitavam de continuidade na TN após a alta hospitalar, a partir de 2004 houve a necessidade de organizar a atenção, sendo designado um nutricionista no nível central para avaliação dos casos e dispensação mais efetiva. Nesse momento, o monitoramento do estado nutricional com dados de peso e altura dos pacientes beneficiados era realizado pela equipe de atenção básica das unidades de saúde (UBS), que enviavam os dados periodicamente à nutricionista no nível central. A partir de então, 
foram definidos alguns critérios mínimos para o fornecimento de FAI, com a padronização das especificações técnicas e nutricionais dos produtos; a definição dos diagnósticos nutricionais; e a realização da análise epidemiológica dos dados, o que culminou na elaboração da primeira versão de um protocolo, inicialmente denominado de Programa de Dietas Enterais e Leites Especiais do Município de Curitiba.

Mesmo com essa organização, foram observadas algumas dificuldades decorrentes da ausência de profissionais capacitados em TN e em alergias e intolerâncias alimentares. As principais dificuldades encontradas pelo nutricionista do nível central foram: distribuição de produtos sem a devida avaliação e acompanhamento específico conforme evolução dos indivíduos, dificuldades na avaliação de doentes acamados; falta de orientação sobre o uso correto dos produtos recebidos, como preparo, higiene, armazenamento e administração; grande variedade de produtos solicitados em relação a marcas e sabores; ausência de orientação alimentar coadjuvante ao uso do produto; insegurança da equipe de saúde em avaliar a suspensão do produto; e, por fim, a sustentabilidade da dispensação dos produtos custeados pelo município.

Durante o período de 2004 a 2005, foi firmada parceria com as instituições de ensino superior (IES) da cidade para implantação de um projeto-piloto com estudantes de Nutrição que fariam a avaliação nutricional dos indivíduos em TND, com o objetivo de embasar a necessidade de organização do serviço de cuidado integral à saúde desses pacientes. Esta parceria possibilitou o diagnóstico da situação dos usuários de TND do município, bem como contribuiu na formação dos acadêmicos das IES, proporcionando o contato com a população.

A partir de 2006, a SMS elaborou a proposta de implantação de um novo programa, com vistas à atenção nutricional no seu sentido mais amplo, ou seja, a atenção integral e não apenas a distribuição de fórmulas alimentares. Tal proposta vai ao encontro dos fundamentos e diretrizes da Política Nacional de Atenção Básica, como acesso universal, integralidade e longitudinalidade do cuidado. ${ }^{7}$ Para colocar em prática esse novo conceito, a SMS incluiu no seu quadro funcional, inicialmente, dez nutricionistas na atenção básica, sendo um em cada Distrito Sanitário e um no nível central, para coordenar as ações relacionadas ao tema, o que possibilitou o atendimento individual de pessoas com necessidades especiais de alimentação.

Considera-se necessidade especial de alimentação quando, devido a alguma disfunção ou doença associada, a pessoa não pode ou não consegue se alimentar normalmente, necessitando de planejamento nutricional específico. Nessa condição enquadram-se crianças com alergia ou intolerância alimentar, doentes com desnutrição secundária devido a doença de base e aqueles com doenças que comprometem o funcionamento normal do trato gastrointestinal que impliquem má absorção e/ou uso de sonda enteral como via de alimentação. Com os novos profissionais apoiando a equipe de atenção básica, foi possível reorganizar o Programa de Dietas Enterais e Leites Especiais, que passou a ser chamado PAN. 


\section{Histórico do PAN - 2006 a 2011}

Com a chegada dos nutricionistas, realizou-se um mutirão para o diagnóstico da situação dos indivíduos atendidos pela SMS, contemplando a avaliação nutricional e utilização dos produtos fornecidos. Neste momento, foram encontrados novos problemas relacionados a: evolução da via alimentar; continuidade do recebimento de dieta enteral para suprir $100 \%$ de alimentação via sonda; recusa de utilização dos produtos fornecidos, existindo estoques remanescentes nos domicílios; utilização incorreta da quantidade dos produtos prescritos e ingestão alimentar incompatível com quadro de alergia alimentar - ou seja, alguns produtos estavam sendo fornecidos sem necessidade.

Com o objetivo de nivelar o conhecimento dos nutricionistas atuantes no PAN, a SMS contratou uma empresa especializada em cursos, edição de livros técnicos e materiais educativos na área da Nutrição, para capacitação dos nutricionistas em TND e elaboração de um manual de cuidados nutricionais. Durante o curso, o manual previamente elaborado pela empresa recebeu contribuições das nutricionistas da SMS, para que fosse adaptado à realidade da atenção básica e assim foi chamado de Manual de Cuidados Nutricionais de Curitiba. O material construído foi a base da capacitação realizada e passaria a servir como material de apoio ao PAN, pois contava com orientações técnicas para o manejo da TND, como vias de acesso de alimentação, categorias de fórmulas alimentares e métodos de avaliação nutricional.

A partir desse manual, foi construído o Protocolo de Cuidados Nutricionais de Curitiba. Como parte do aprimoramento profissional, também se realizou parceria com o Departamento de Nutrição da Universidade Federal do Paraná, juntamente com o Centro Colaborador de Alimentação e Nutrição da Região Sul (CECAN-Sul), para educação continuada com os mais variados temas, como insuficiência renal, câncer, doenças neurológicas, disfagia, alergia à proteína do leite de vaca, diabetes, aulas de técnica dietética em laboratório de nutrição para a elaboração e preparo de preparações não industrializadas.

As fórmulas não industrializadas são utilizadas principalmente para usuários estáveis clinicamente, com doenças crônicas ou em tratamento paliativo, não necessariamente na sua terminalidade, que necessitam de TND. No Brasil, as duas modalidades de fórmulas, industrializadas e com alimentos, são utilizadas no domicílio; cada uma tem sua especificidade, conforme o diagnóstico clínico, prognóstico, perfil nutricional e ainda situação socioeconômica da família. Para a atuação dos nutricionistas, foram adquiridos equipamentos como balanças portáteis, plicômetros, estadiômetros e fitas métricas, adquiridos com recursos financeiros provenientes da SMS de Curitiba e do Governo Federal. 
Juntamente com as capacitações, treinamento de abordagem ao usuário e avaliação nutricional, a equipe de nutricionistas da SMS interagiu com sua prática, contribuindo para a sistematização do serviço no PAN com a padronização dos fluxos, normas e condutas. Nesse período aconteceram aproximações com os serviços de atenção especializada ambulatorial e hospitalar do município, com o objetivo de integrar as práticas de cuidado que os indivíduos recebiam nos diferentes pontos de atenção à saúde.

Com o aumento do número de casos de crianças com alergia à proteína do leite de vaca (APLV), a SMS percebeu a importância de ter no seu quadro funcional um profissional especializado para seu atendimento. Sendo assim, em julho de 2007, incorporou um pediatra especialista em gastroenterologia para atender especificamente aos casos de APLV e intolerância a lactose do PAN. Em 2009, com a implantação dos Núcleos de Apoio à Saúde da Família (NASF) no município, o quadro de nutricionistas foi ampliado para 29 profissionais. Posteriormente, em outubro de 2013, mais um profissional especialista em alergia se integrou à equipe, para atender a toda a demanda do programa.

\section{Implementação do Protocolo: uma conquista histórica}

Entre os anos de 2006 a 2011, o trabalho de sistematização e organização do serviço foi sendo aprimorado, até que em abril de 2011, o Protocolo foi oficialmente lançado e publicado, sendo validado por diversas sociedades científicas. ${ }^{9}$

Como resultados do PAN, é possível destacar a assistência multiprofissional integrada desenvolvendo importante papel no suporte clínico e emocional do paciente e da família; a humanização do tratamento, por meio do atendimento domiciliar, proporcionando maior conforto e segurança ao paciente e à família; a realização de ações periódicas de assistência à saúde, proporcionando maior segurança no tratamento por meio do monitoramento e intervenções, quando necessário; a avaliação individualizada de acordo com as necessidades de cada paciente; a realização do plano alimentar adaptado, conforme hábitos e cultura da família; a evolução no tratamento devido ao monitoramento e orientações periódicas; atendimento de maior número de usuários e reforço do trabalho intersetorial com as diversas secretarias e órgãos do município.

Além do Protocolo, outros materiais foram desenvolvidos pela SMS e auxiliam no desenvolvimento das atividades relacionadas ao PAN, como a Cartilha de Terapia Nutricional Enteral Domiciliar (TNED), a Cartilha de Alimentação Infantil ${ }^{10}$ e ainda a Cartilha de Alimentação para uma Vida Saudável, disponíveis no site da SMS de Curitiba. ${ }^{11}$ 
A implantação do PAN vem ao encontro do proposto na Política Nacional de Alimentação e Nutrição (PNAN), ${ }^{1}$ onde é colocada a necessidade de melhor organização dos serviços de saúde do país no que se refere às principais demandas geradas pelos agravos relacionados à má alimentação como desnutrição, obesidade, carências nutricionais específicas e doenças crônicas não transmissíveis. Inclui, no entanto, também no rol de demandas para a atenção nutricional no SUS, o cuidado aos indivíduos portadores de necessidades alimentares especiais (PNAN, 2012).

A figura 1 apresenta a linha do tempo em relação às ações voltadas à atenção de pessoas com necessidades especiais de alimentação na SMS de Curitiba.

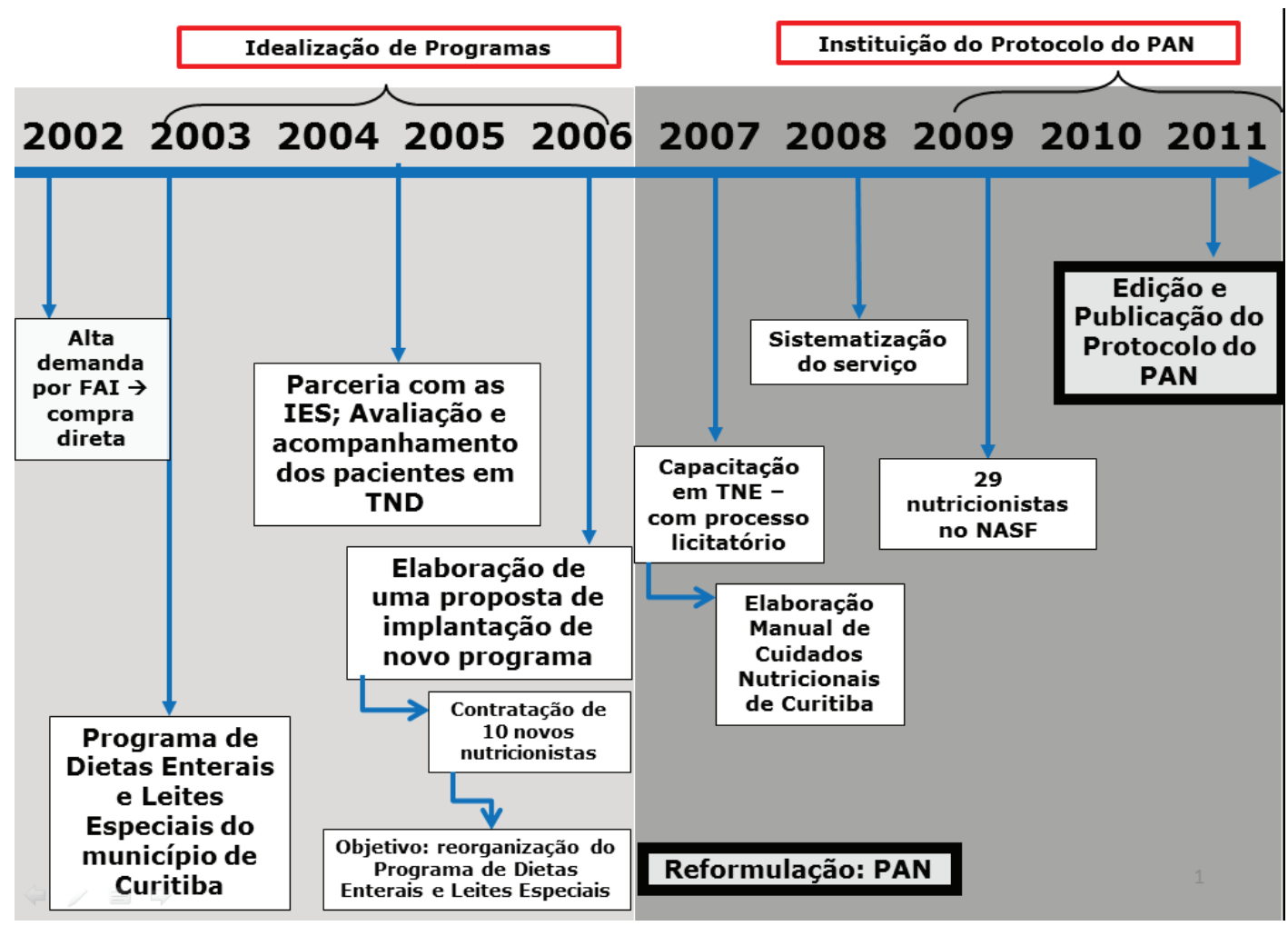

Figura 1. Marco histórico da implementação do Programa de Atenção Nutricional. CuritibaPR, 2002 a 2011.

Notas: FAI: fórmulas alimentares industrializadas; IES: Instituições de Ensino Superior; TND: Terapia Nutricional Domiciliar; TNE: Terapia Nutricional Enteral; NASF: Núcleo de Apoio à Saúde da Família; PAN: Programa de Atenção Nutricional às Pessoas com Necessidades Especiais de Alimentação. 


\section{Ontem, hoje e amanhã̃: avanços e perspectivas para o PAN}

Desde a implementação do PAN em 2006 até dezembro de 2013, foram atendidos 5.173 pacientes. O número de atendimentos por ano passou de 647 em 2006, para 1.529 em 2013. O atendimento, como já mencionado, visa principalmente à atenção nutricional que vai além da distribuição de FAI.

Conforme análise dos dados dos pacientes incluídos no PAN em dezembro de 2013, dos 785 pacientes em continuidade no programa, 35,3\% eram atendidos com orientação nutricional e acompanhamento pela equipe da UBS, sem o fornecimento de FAI. O atendimento do PAN contempla: crianças $(50,3 \%)$, idosos $(25,5 \%)$, adultos $(14,8 \%)$ e adolescentes $(9,4 \%)$. Dos pacientes atendidos, $57,7 \%$ se deslocam até a UBS e 42,3\% permanecem no domicílio, devido a dificuldades de locomoção, embora todos os pacientes recebam ao menos uma visita domiciliar do nutricionista, para conhecimento das condições e modo de vida da família.

Em relação aos grupos de doenças comuns das pessoas atendidas no PAN, a que lidera é a APLV (30,3\%), seguida das doenças do sistema nervoso central, principalmente paralisia cerebral e demências (24\%), e câncer (11,2\%), doenças do aparelho circulatório, principalmente acidente vascular encefálico $(9,9 \%)$, doença renal, principalmente crônica $(2,2 \%)$, e outros diagnósticos $(22,4 \%)$.

A via de acesso alimentar das pessoas atendidas no PAN é a oral (56,3\%), seguida por alimentação via sonda (37,7\%) e via mista, oral associada a sonda (6\%). Dos 37,7\% pacientes com alimentação via sonda enteral, o que predomina é a gastrostomia (69,9\%).

Com demandas crescentes no atendimento às pessoas com necessidades alimentares especiais na atenção básica, há necessidade de avanço e aprimoramento das ações e na operacionalização do PAN, adequando-as conforme as necessidades dos usuários e aspectos setoriais e intersetoriais. Neste sentido, é muito importante a maior aproximação com a atenção ambulatorial e especializada de saúde, visando fortalecer o trabalho em rede e a melhor atenção à saúde desses indivíduos. Diante do exposto, é essencial a revisão periódica e sistemática do protocolo do programa. A educação permanente é outro desafio para que se mantenham a formação e atualização dos profissionais para o atendimento dos pacientes em TND.

A organização do PAN constitui um marco importante para o SUS, e espera-se que o trabalho aqui apresentado possa fomentar estudos posteriores, já que são escassas as referências de protocolos do cuidado em TND na atenção básica à saúde. 


\section{Considerações finais}

O marco histórico do PAN demonstrou que houve avanços na qualidade de assistência aos pacientes com necessidades alimentares especiais. Construir o protocolo de cuidado garantiu a sustentabilidade do programa, promoveu maior envolvimento e satisfação/segurança dos profissionais envolvidos, mas especialmente, cumpriu com o objetivo da proposta de melhorar significativamente a qualidade do cuidado ao usuário.

\section{Agradecimentos}

A Danielle Rodrigues Lecheta, nutricionista responsável pela sistematização do protocolo do PAN na SMS de Curitiba, e em nome da nutricionista Cristiane Aparecida Azevedo de Assis Machado, agradecemos a todas as demais nutricionistas que auxiliaram na construção do protocolo do PAN, bem como a todas aquelas que o operacionalizam nas US da SMS de Curitiba.

\section{Referências}

1. Brasil. Ministério da Saúde. Política Nacional de Alimentação e Nutrição. Secretaria de Atenção à Saúde. Brasília: Ministério da Saúde; 2012.

2. Brasil. Constituição Federal. Artigo 196. Brasília: Senado Federal; 1988.

3. Brasil. Lei no 8.080, de 19 de setembro de 1990. Dispõe sobre as condições para a promoção, proteção e recuperação da saúde, a organização e o funcionamento dos serviços correspondentes e dá outras providências. Diário Oficial da União 20 set 1990. Brasília; 1990.

4. Brasil. Ministério das Relações Exteriores. A segurança alimentar e nutricional e o direito humano à alimentação no Brasil. Documento elaborado para a visita ao Brasil do Relator Especial da Comissão de Direitos Humanos da Organização das Nações Unidas sobre Direito à Alimentação. Brasília: Ipea; 2002.

5. Brasil. Resolução RDC no 63, de 06 de julho de 2000. Aprova o Regulamento Técnico para fixar os requisitos mínimos exigidos para a Terapia de Nutrição Enteral. Diário Oficial da União 07 jul. 2000.

6. Anker SD, John M, Pedersen PU, Raguso C, Cicoira M, Dardai E, et al. ESPEN guidelines on enteral nutrition: cardiology and pulmonology. Clin. Nutr. 2006; 25(2):311-8.

7. Brasil. Ministério da Saúde. Política nacional de atenção básica. Brasília: Ministério da Saúde; 2012.

8. Brasil. Portaria no 120 de 14 de abril de 2009, Assistência de Alta Complexidade de Terapia Nutricional. [Internet]. [acesso em 02 jun. 2014]. Disponível em: http://bvsms.saude.gov.br/bvs/saudelegis/ sas/2009/prt0120_14_04_2009.html 
9. Curitiba. Secretaria Municipal da Saúde. Programa de atenção nutricional às pessoas com necessidades especiais de alimentação (PAN) [Internet]. Curitiba: Coordenação de Alimentação e Nutrição; 2011. [acesso em 02 jun. 2014]. Disponível em: http://saude.curitiba.pr.gov.br/images/programas/arquivos/ alimentacao/alimentacao_003.pdf

10. Curitiba. Secretaria Municipal da Saúde. Cartilha alimentação infantil [Internet]. Curitiba: Coordenação de Alimentação e Nutrição; 2007. [acesso em 02 jun. 2014]. Disponível em: http://189.28.128.100/ nutricao/docs/evento/ii_forum_edu_an/alimentacao_infantil_cristiane_machado.pdf

11. Curitiba. Secretaria Municipal da Saúde. Cartilha alimentação para uma vida saudável [Internet]. Curitiba: Coordenação de Alimentação e Nutrição; 2009. [acesso em 02 jun. 2014]. Disponível em: http://www.crn8.org.br/audiovisual/publicacoes/2010/alimentacao-para-uma-vida-saudavel/ Alimentacao_para_uma_vida_saudavel.pdf

Recebido: $15 / 4 / 2014$

Revisado: 29/5/2014

Aprovado: 07/7/2014 